\title{
Opto-acoustic Coupling and Brillouin Phenomena in Microstructure Optical Fibers
}

\author{
Hervé Maillotte $^{(1)}$, Jean-Charles Beugnot ${ }^{(1)}$, Birgit Stiller ${ }^{(1)}$, Min Won Lee ${ }^{(1)}$, Duc Minh Nguyen ${ }^{(1)}$, \\ Michael Delqué( ${ }^{(1)}$, Sarah Benchabane ${ }^{(2)}$, Vincent Laude ${ }^{(2)}$, Stella Foaleng Mafang ${ }^{(3)}$, Luc Thévenaz ${ }^{(3)}$, \\ Géraud Bouwmans $^{(4)}$, Alexandre Kudlinski ${ }^{(4)}$, Gilles Mélin ${ }^{(5)}$, Jérôme Hauden ${ }^{(6)}$, Thibaut Sylvestre ${ }^{(1)}$ \\ ${ }^{(1)}$ P.M. Duffieux Optics Department, ${ }^{(2)}$ Micro-Nano-Sciences and Systems Department, FEMTO-ST Institute, \\ UMR 6174 CNRS-Université de Franche-Comté, 16 Route de Gray, 25030 Besançon cedex, France \\ ${ }^{(3)}$ Group for Fibre Optics - Institute of Electrical Engineering, EPFL Swiss Federal Institute of Technology, Station 11, CH- \\ 1015 Lausanne, Switzerland \\ (4) Laboratoire PhLAM, IRCICA, UMR 8523 CNRS-Université des Sciences et Technologies de Lille, 59655 Villeneuve \\ d'Ascq, France \\ ${ }^{(5)}$ Draka, Route de Nozay, 91460 Marcoussis, France \\ ${ }^{(6)}$ Photline Technologies, 16 rue Jouchoux, 25000 Besançon, France
}

Email: herve.maillotte@univ-fcomte.fr

\begin{abstract}
Like photonic crystals have revolutionized the way of manipulating optical waves at the sub-micron scale, phononic crystals have more recently played similar decisive role for sound waves, or more generally elastic waves. Then, the idea of coupling light and sound in purposely designed microstructures is now emerging. In this respect, the periodic, wavelength-scale (for both optic and high-frequency acoustic waves) transverse air-hole microstructure of photonic crystal fibers (PCFs) provides additional degrees of freedom for light-sound interactions. PCFs can indeed exhibit photonic and phononic bandgap effects, allowing for tight confinement and joint waveguiding of both types of waves [1]. Electrostriction-driven Brillouin phenomena, namely backward Stimulated Brillouin Scattering (SBS) and forward Guided Acoustic Wave Brillouin Scattering (GAWBS), constitute an important category of such optoacoustic coupling. The geometry of PCFs can dramatically modify the Brillouin spectrum, the gain and the stimulated Brillouin threshold, globally yielding much richer opto-acoustic dynamics and spectral features than in conventional fibers [2-11]. Specific transverse or longitudinal guided acoustics modes in the $100 \mathrm{MHz}-10$ $\mathrm{GHz}$ range can thus be selectively excited, resonantly enhanced and tightly confined within the microstructure, with an intimate dependence on its $\mu \mathrm{m}$ or sub- $\mu \mathrm{m}$ geometry. All these specific features have great potential for developing novel PCF-based distributed Brillouin sensors [6,7,12-14], for high-resolution longitudinal mapping of the intrinsic fluctuations of the fiber microstructure [15], and more generally for developing original tools of optical signal processing [1$4,9,16,17]$. This talk will give a comprehensive overview of these original behaviors in a range of PCFs.
\end{abstract}

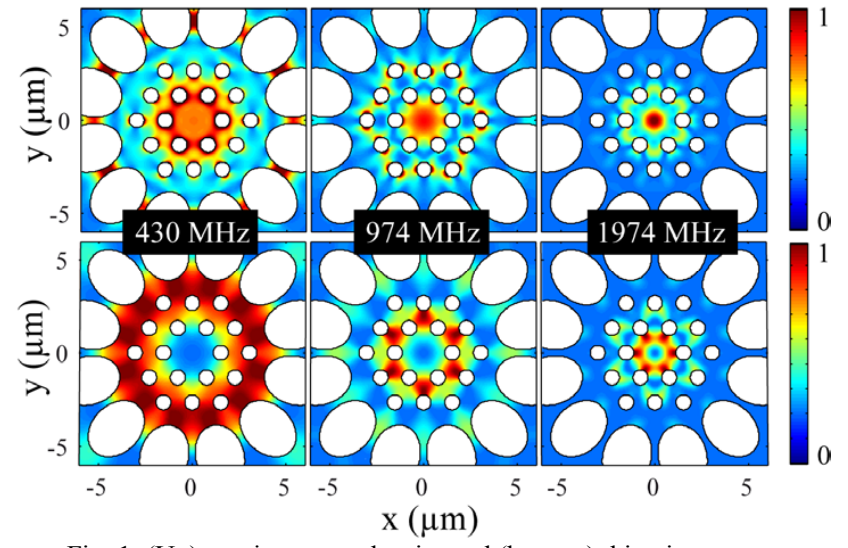

Fig. 1. (Up): strain energy density and (bottom): kinetic energy distributions calculated by full vector finite element method, showing the frequency-selective excitation of guided acoustic transverse modes in a multi-scale microstructure fiber.

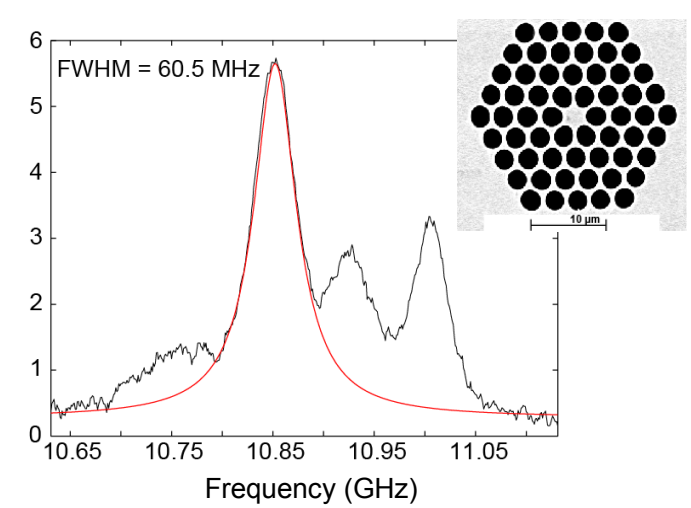

Fig. 2. Multi-peaked stimulated backward Brillouin scattering spectrum in a solid core photonic crystal fiber.

This work is supported by the Fond Européen de Développement Régional (FEDER), French-Swiss Interreg IVA program under project CD-FOM. 


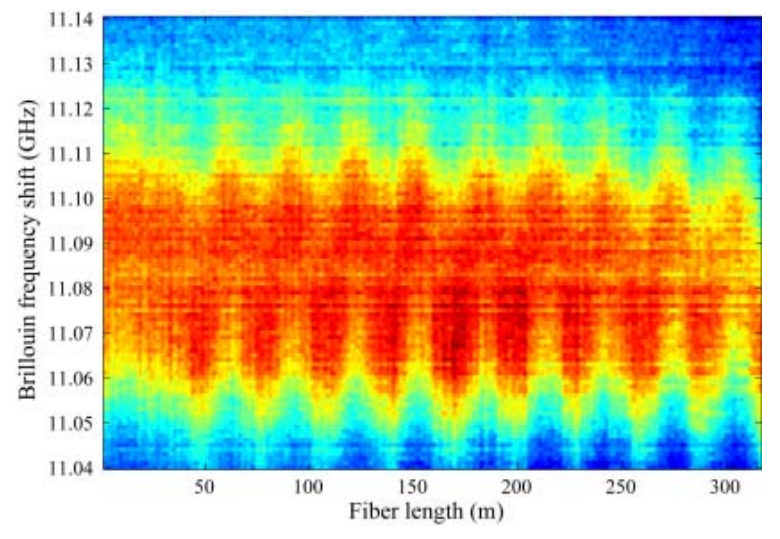

Fig. 3. Brillouin Echo Distributed Sensing measurement: Longitudinal mapping of the Brillouin gain spectrum in a periodically-varying microstructure fiber for SBS mitigation.

\section{REFERENCES}

[1] V. Laude, A. Khelif, S. Benchabane, M. Wilm, T. Sylvestre, B. Kibler, A. Mussot, J.M. Dudley, H. Maillotte, "Phononic bandgap guidance of acoustic modes in photonic crystal fibers", Phys. Rev. B, vol. 71, pp. 045107, January 2005.

[2] P. Dainese, P.St.J. Russell, N. Joly, J.C. Knight, G.S. Wiederhecker, H.L. Fragnito, V. Laude, A. Khelif, "Stimulated Brillouin scattering from multi-GHz-guided acoustic phonons in nanostructured photonic crystal fibres", Nat. Phys., vol. 2, pp. 388-392, June 2006.

[3] P. Dainese, P.St.J. Russell, G.S. Wiederhecker, N. Joly, H.L. Fragnito, V. Laude, A. Khelif, "Raman-like light scattering from acoustic phonons in photonic crystal fiber", Opt. Ex., vol. 14, pp. 4141-4150, May 2006.

[4] D. Elser, U.L. Andersen, A. Korn, O. Gloeckl, S. Lorenz, C. Marquardt, G. Leuchs, "Reduction of guided acoustic wave Brillouin scattering in photonic crystal fibers", Phys. Rev. Lett., vol. 97, pp. 133901, September 2006.

[5] J.C. Beugnot, T. Sylvestre, H. Maillotte, G. Mélin, V. Laude, "Guided acoustic wave Brillouin scattering in photonic crystal fibers", Opt. Lett., vol. 32, pp. 17-19, January 2007.

[6] J.C. Beugnot, T. Sylvestre, D. Alasia, H. Maillotte, V. Laude, A. Monteville, L. Provino, N. Traynor, S. Foaleng Mafang, L. Thévenaz, "Complete experimental characterization of stimulated Brillouin scattering in photonic crystal fiber ", Opt. Ex., vol. 15, pp. 1551715522, November 2007.

[7] A. Minardo, R. Bernini, W. Urbanczyk, J. Wojcik, N. Gorbatov, M. Tur, L. Zeni, "Stimulated Brillouin scattering in highly birefringent microstructure fiber: experimental analysis", Opt. Lett., vol. 33, pp. 2329-2331, October 2008.

[8] J.E. McElhenny, R.K. Pattnaik, J. Toulouse, K. Saitoh, M. Koshiba, "Unique characteristic features of stimulated Brillouin scattering in small-core photonic crystal fibers", J. Opt. Soc. Am. B, vol. 25, pp. 582-593, April 2008.

[9] M.S. Kang, A. Nazarkin, A. Brenn, P.St.J. Russell, "Tightly trapped acoustic phonons in photonic crystal fibres as highly nonlinear artificial Raman oscillators", Nat. Phys., vol. 5, pp. 276-280, April 2009.

[10] J.C. Beugnot, B. Stiller, S. Foaleng Mafang, M.W. Lee, M. Delqué, A. Kudlinski, H. Maillotte, V. Laude, L. Thévenaz, T. Sylvestre, "Experimental Observation of Brillouin Linewidth Broadening and Decay Time in Photonic Crystal Fiber", Proc. of 36th European
Conference and Exhibition on Optical Communication (ECOC 2010), Torino (Italy), Sep. 2010, paper Tu.4.D.5.

[11] B. Stiller, M. Delqué, J.C. Beugnot, M.W. Lee, G. Mélin, H. Maillotte, V. Laude, T. Sylvestre, "Frequency-selective excitation of guided acoustic modes in a photonic crystal fiber", Opt. Ex., vol. 19, pp. 7689-7694, April 2011.

[12] L.F. Zou, X.Y. Bao, V.S. Afshar, L. Chen, "Dependence of the Brillouin frequency shift on strain and temperature in a photonic crystal fiber", Opt. Lett., vol. 29, pp. 1485-1487, July 2004.

[13] E. Carry, J.C. Beugnot, B. Stiller, M.W. Lee, H. Maillotte, T. Sylvestre, "Temperature coefficient of the highfrequency guided acoustic mode in a photonic crystal fiber", Appl. Opt., vol. 50, pp. 6543-6547, December 2011.

[14] M.W. Lee, B. Stiller, J. Hauden, H. Maillotte, C. Roch, L. Thévenaz, T. Sylvestre, "Differential Phase-Shift-Keying Technique-Based Brillouin Echo-Distributed Sensing", IEEE Photonics Technol. Lett., vol. 24, pp. 79-81, January 2012.

[15] B. Stiller, S. Foaleng-Mafang, J.C. Beugnot, M.W. Lee, M. Delqué, G. Bouwmans A. Kudlinski, L. Thévenaz, H. Maillotte, T. Sylvestre, "Photonic crystal fiber mapping using Brillouin echoes distributed sensing", Opt. Ex., vol. 18, pp. 20136-20142, September 2010.

[16] J. Spring, B. Ward, "Brillouin gain suppression in photonic crystal fibers with random acoustically microstructured cores", Opt. Ex., vol. 35, pp. 31-33, January 2010.

[17] B. Stiller, A. Kudlinski, M.W. Lee, G. Bouwmans, M. Delqué, J.C. Beugnot, H. Maillotte, T. Sylvestre, "SBS Mitigation in a Microstructured Optical Fiber by Periodically Varying the Core Diameter, IEEE Photonics Technol. Lett. (2012), in press. 